\title{
Effect of Decompression on Jaw Cystic Lesions Based on Three-Dimensional Volumetric Analysis
}

\author{
Yeh-Jin Kwon ${ }^{1}$, Kyeong-Soo Ko ${ }^{1}$, Byung-Kyu So ${ }^{1}$, Dong-Hyuck Kim ${ }^{1}$, Hyon-Seok Jang ${ }^{2}$, \\ Soo-Ho Kim ${ }^{3}$ D, Eui-Seok Lee ${ }^{1, *}$ and Ho-Kyung Lim ${ }^{1, * \mathbb{C}}$ \\ 1 Department of Oral and Maxillofacial Surgery, Korea University Guro Hospital, Seoul 08308, Korea; \\ yehjin51@nate.com (Y.-J.K.); k1015ks@naver.com (K.-S.K.); sobk4728@naver.com (B.-K.S.); \\ hyperlion@naver.com (D.-H.K.) \\ 2 Department of Oral and Maxillofacial Surgery, Korea University Ansan Hospital, Gyeonggi-do 15355, Korea; \\ omfs1109@korea.ac.kr \\ 3 Department of Oral and Maxillofacial Surgery, Chungnam National University Hospital, Daejeon 35015, \\ Korea; patron12@naver.com \\ * Correspondence: ees225@hanmail.net (E.-S.L.); ungassi@naver.com (H.-K.L.); Tel.: +82-10-3367-8671 (E.-S.L.); \\ +82-10-6253-0872 (H.-K.L.); Fax: +82-2-837-6245 (E.-S.L.); +82-2-837-6245 (H.-K.L.)
}

Received: 21 October 2020; Accepted: 6 November 2020; Published: 10 November 2020

\begin{abstract}
Background and objectives: This study aimed to evaluate the effectiveness of decompression on jaw cysts according to various parameters by volumetric analysis using three-dimensional computed tomography. Materials and methods: Fifty patients who underwent surgical decompression of the jaw cystic lesion were selected, and their preoperative and postoperative computed tomography results between 3 and 27 months were collected. Volumetric analysis was performed to evaluate any differences in the rate of volumetric change according to the sex, age, initial volume of the lesion, duration, location of the lesion, tooth extraction, expansion of the cortical layer, and pathological diagnosis. Multiple linear regression and generalised linear mixed models were used for statistical analyses. Results: The mean reduction rate among all patients was $54.68 \%$. Multiple linear regression analysis revealed that higher reduction rates were associated with a long decompression period, young patient age, and location of the cyst in the posterior maxilla. Generalised linear mixed models revealed that higher reduction rates were associated with a long decompression period and young patient age. Conclusions: Decompression was an effective treatment for reducing the cyst size in all patients. Its effectiveness increased with a long treatment duration, young patient age, and cyst location in the posterior maxilla three-dimensionally.
\end{abstract}

Keywords: surgical decompression; cyst; 3D imaging; volume

\section{Introduction}

Cystic lesions are one of the most common pathologies in the oral and maxillofacial area. These lesions are more frequently observed in the upper and lower jaws than other bones in the human body because a cell remnant of the embryonic neuroectoderm can trigger pathogenesis [1,2]. Standard treatment methods for cystic lesions are enucleation, which usually removes cystic walls at once, marsupialisation, which connects to the surrounding oral mucous membranes, and decompression, which seeks to form a bone by relieving pressure in the lesion [1,2]. To determine the management methods of cystic lesions, surgeons consider the size, location, pathological diagnosis, and anatomical structures of the lesions. If the lesion invades the adjacent structures, or if the primary enucleation could induce the pathological fracture or neurological damage, marsupialisation or decompression is initially considered [3].

Decompression is a technique that reduces the pressure of the cystic fluid in the cystic cavity and induces bone deposition towards the cystic wall $[3,4]$. If the size of the lesion is decreased after the 
decompression surgery and the condition of the lesion does not affect the adjacent important anatomical structures such as the inferior alveolar nerve and teeth around the lesion, the timing of cyst enucleation is determined based on the surgeons' judgment [5,6]. In previous studies, the factors affecting cyst volume reduction were controversial, and the methods of analysis of volumetric changes varied. Moreover, several reports regarding the volumetric analysis of cysts used panoramic radiography with morphological distortion $[7,8]$. To overcome some of the limitations of panoramic radiography, we have tried to use computed tomography (CT) analysis to determine the three-dimensional (3D) volumetric changes in this study.

This study aimed to investigate the efficacy of decompression and analyse the factors affecting the volumetric change of cystic lesions based on sex, age, total duration of decompression, initial volume of the lesion, location of the lesion, presence of involved teeth, cortical bone expansion, and specific pathological diagnosis.

\section{Materials and Methods}

\subsection{Patients' Criteria}

The study was conducted retrospectively on patients who underwent cystic decompression surgery in their jaws at the Department of Oral and Maxillofacial Surgery of Korea University Guro Hospital from 2012 to 2020. The inclusion criteria were as follows: patients who were diagnosed with cyst or cystic benign tumour histopathologically, patients who underwent decompression surgery based on surgeons' decision, and patients who underwent preoperative and postoperative CT at least once. The exclusion criteria were as follows: patients who did not visit the hospital after undergoing decompression surgery, patients with omitted CT data, patients with a vanished drain during the follow-up periods, and patients having other accompanying pathologies such as sinusitis, which could affect volumetric changes, and osteoporosis, which could decrease the bone deposition ability. A total of 50 patients met the inclusion criteria and were subsequently included in our study. This study was approved by the Korea University Guro Hospital Ethics Committee (Approval No: 2020GR0450, Approval date: 7 October 2020). In addition, the clinical trial was in accordance with certification for ISO14155.

\subsection{Surgical Procedures for Decompression}

Surgical procedures of all patients were performed under the standardised protocol as follows. Decompression was performed under local anaesthesia with lidocaine, including 1:100,000 epinephrine (Yuhan Corporation, Seoul, Korea) around the lesion, accompanied by minimal incision using a \#15 scalpel. If the cortical bone of the incision area was intact, an access hole was formed using high-speed handpiece and round bur. The hole size was $1 \mathrm{~mm}$ larger than the diameter of the tube. After approaching the cyst, a part of the cystic membrane tissue was incised and fixed with formalin solution for the histological confirmation. After inserting the Penrose drain into the cyst, the drain was sutured on the oral mucosa using the 4-0 Ethilon nylon (Ethicon, Georgia, MI, USA). The drain was cut $0.5 \mathrm{~mm}$ from the mucosa. After suturing, irrigation was performed several times with normal saline using a tube. After the operation, all patients were prescribed cefaclor hydrate $250 \mathrm{mg}$ (thrice a day), aceclofenac $100 \mathrm{mg}$ (twice a day), and esomeprazole $20 \mathrm{mg}$ orally (once a day), and a $0.2 \%$ chlorhexidine mouthwash was topically rinsed (for $1 \mathrm{~min}$, thrice a day) for 7 days. For patients with allergy or taking other drugs, replaced prescription was made in consideration of its interaction with the other drugs. If a third molar or another tooth was involved with the cyst, extraction was initially performed, and the insertion of a tube was performed consecutively. Patients were trained to irrigate themselves with sterile saline. After the decompression surgery, the surgeon assessed the drain condition monthly. If the drain was contaminated, it was replaced with a new one. 


\subsection{Computed Tomography Data Acquisition}

3D CT data using the cone beam CT device (Vatech, Seoul, Korea) or medical CT device (Siemens Definition Edge, Erlangen, Germany) were obtained. A cut of at least a 1-mm slice was obtained from all CTs. All patients underwent CT before surgery and once or several times after surgery between 3 and 27 months.

\subsection{D Volume Measurements}

The open-source software ITK-SNAP (Penn Image Computing and Science Laboratory, PA, USA) was used to measure the volume of the cystic lesion. The CT digital imaging and communications in medicine (DICOM) datasets were imported into the program and were sliced in the axial plane. In setting the cystic lesion margin, Hounsfield unit (HU) values of the 3D voxel were designated in slice. We assumed that the cystic cavity had the value of the lower threshold range from -1000 to $-500 \mathrm{HU}$ and the upper threshold range from 500 to $1000 \mathrm{HU}$ in CT slice. When the surface boundary of the intra-bony cyst bone was clear, semi-automatic segmentation, a preprogrammed automatic system was performed. However, when the surface boundary line was unclear, manual segmentation was performed. After the designation of the cystic area in each slice, a 3D volumetric image was rendered by the sum of each slice in axial plane (Figure 1a), and 3D cystic volume was measured and calculated (Figure 1b). In cases involving teeth, to remove the volume of teeth, the volume of extraction socket was excluded in the total volume. Comparing between preoperative and postoperative volume measurements, the percentage reduction in the volume (PRV) of cystic lesions in the jaw was calculated as follows:

$$
\operatorname{PRV}(\%)=\frac{\text { (Cystic Volume before Decompression-Cystic Volume after Decompression })}{\text { Cystic Volume before Decompression }} \times 100
$$

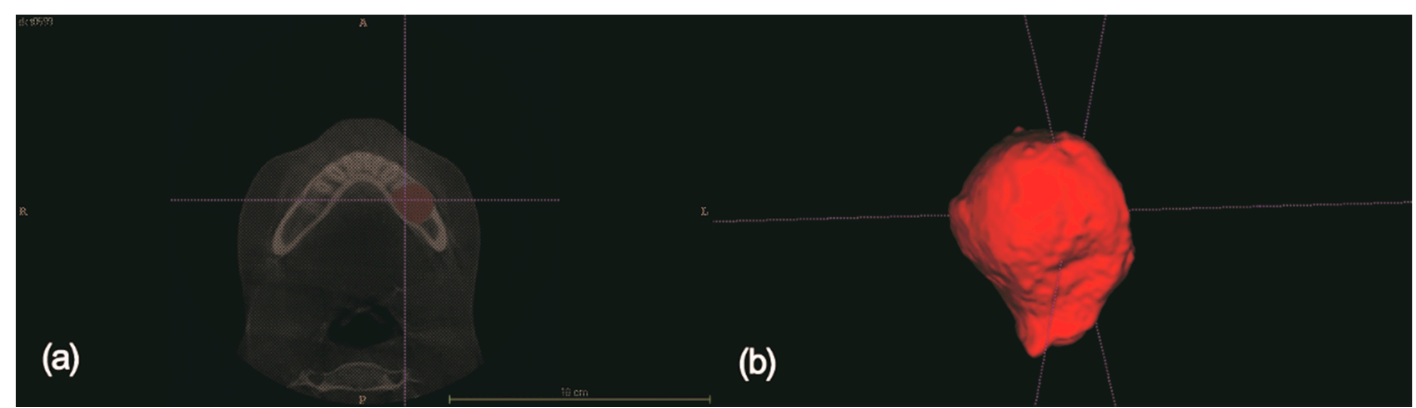

Figure 1. (a) Segmentation of the cystic lesion in axial plane of computed tomographic data.

(b) Computerized three-dimensional reconstruction of the cystic lesion. Volume was measured with ITK-SNAP (Penn Image Computing and Science Laboratory, PA, USA).

\subsection{Determination of Effective Parameters}

We assumed that the following parameters could influence the effect of decompression: sex, age, total duration of decompression, initial volume of the lesion, location of the lesion, presence of involved teeth, cortical bone expansion, and a specific pathological diagnosis.

Considering the low resistance to expansion pressure of the maxillary sinus, it was classified into three areas based on the anatomical location (anterior maxilla, posterior maxilla, and mandible). Considering the degree of cortical layer expansion, the criterion was also based on the results of the study by Jeong et al. [3]. Cortical layers expanding greater than 1.5 times buccolingually than the opposite normal jaw bone were classified as severe, while those expanding less than 1.5 times were classified as mild. Based on the pathological diagnosis, cysts were divided into the following three groups: dentigerous cyst, radicular cyst, and odontogenic keratocyst. The clinical information from the enrolled patients was investigated. 


\subsection{Statistical Analyses}

To determine the factors affecting PRV, two statistical analysis methods were used in this study. First, to correct the disturbance among factors influencing each other, multiple linear regression analysis was performed with only two images: the pre-decompression image and the final image acquired just before enucleation. Second, to correct the disturbance in factors caused by irregular CT scan timings, a generalised linear mixed model was used, including all images acquired during the complete follow-up process. All statistical analyses were performed with Statistical Analysis System (SAS) version 9.4 (SAS Institute, Cary, NC, USA). The significance level was set to $p<0.05$.

\section{Results}

\subsection{Patient Distribution}

Patients were classified according to sex, age, initial volume of the lesion, duration, location of the lesion, presence of involved teeth, cortical bone expansion, and pathological diagnosis (Table 1). The total number of samples was 50 (37 male and 13 female), with an average age of 32.3 years (range, 7-71 years). The average initial cyst volume was $7.27 \mathrm{~mL}$ (range, $0.77-36.65 \mathrm{~mL}$ ). The mean duration of decompression was 9.41 months (range, 3-27 months). The cystic lesions were located in the anterior maxilla, posterior maxilla, and mandible in 14, 7, and 29 patients, respectively. The number of cases involving the teeth was 12. The numbers of patients with mild and severe cortical expansions were 16 and 34, respectively. The pathological diagnoses were as follows: 17 dentigerous cysts, 26 radicular cysts, and 7 odontogenic keratocysts (Table 1).

Table 1. Percentage reduction in volume of 50 cysts according to parameters *.

\begin{tabular}{|c|c|c|c|c|}
\hline \multirow{2}{*}{ Parameter } & & \multirow{2}{*}{ Number } & \multicolumn{2}{|c|}{ PRV (\%) } \\
\hline & & & Mean & SD \\
\hline \multirow{2}{*}{ Sex } & Male & 37 & 55.812 & 23.72 \\
\hline & Female & 13 & 51.469 & 29.83 \\
\hline \multirow{3}{*}{ Location } & Mandible & 29 & 52.006 & 23.95 \\
\hline & Anterior maxilla & 14 & 53.943 & 29.52 \\
\hline & Posterior maxilla & 7 & 67.254 & 20.05 \\
\hline \multirow{2}{*}{ Tooth extraction } & Extraction & 12 & 55.491 & 26.06 \\
\hline & No extraction & 38 & 54.428 & 25.28 \\
\hline \multirow{2}{*}{ Expansion of the cortical layer } & Mild & 16 & 60.826 & 23.36 \\
\hline & Severe & 34 & 51.792 & 25.85 \\
\hline \multirow{3}{*}{ Pathological diagnosis } & $\mathrm{DC}$ & 17 & 55.272 & 25.24 \\
\hline & $\mathrm{RC}$ & 26 & 55.192 & 25.27 \\
\hline & OKC & 7 & 51.362 & 28.52 \\
\hline Total & & 50 & 54.683 & 25.20 \\
\hline
\end{tabular}

PRV, percentage reduction in volume; SD, standard deviation; DC, dentigerous cyst; RC, radicular cyst; OKC, odontogenic keratocyst; * Age, initial cystic volume, and duration were excluded in the table since these factors were not categorical but numerical data.

All patients showed a reduction in the cyst volume. The average decompression period was 9.41 months. The duration for decompression varied from at least 3 months to a maximum of 27 months, and the value of PRV varied from up to $77.6 \%$ to at least $33.9 \%$.

\subsection{Effective Factors in the Two Statistical Analyses}

The linear multiple regression analysis revealed significant differences in reduction rates with the patient age and duration and location of the lesion. Higher reduction rates were found with the young patient age $(p=0.037)$, long duration of decompression $(p<0.0001)$, and cyst location in the posterior 
maxilla $(p=0.0081)$. Generalised linear mixed models revealed significant differences in reduction rates with the patient age and duration of the lesion. Higher reduction rates were found with the young patient age $(p=0.0124)$ and long duration of decompression $(p=0.0016$; Figure 2 ; Table 2$)$. The regression equation derived from the two analysis methods is as follows:

$$
\mathrm{y}=56.3067+1.3396 \times \text { duration }-0.4376 \times \text { age }
$$
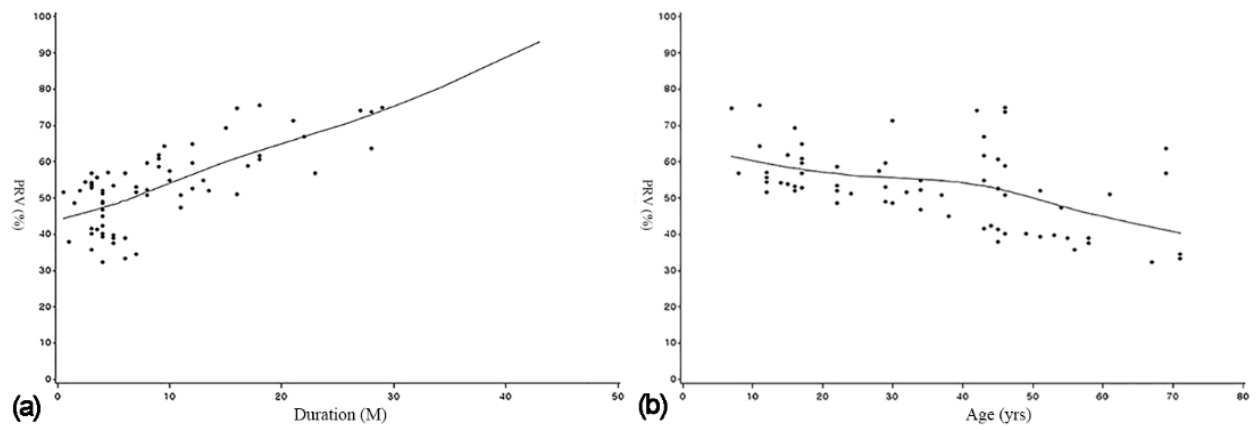

Figure 2. (a) Regression graph between the independent variable duration and the dependent variable PRV. (b) Regression graph between the independent variable age and the dependent variable PRV.

Table 2. Comparison of 50 cystic cases with two statistical methods.

\begin{tabular}{ccc}
\hline Parameter & $\begin{array}{c}p \text {-Value with the Multiple } \\
\text { Linear Regression Analysis }\end{array}$ & $\begin{array}{c}p \text {-Value with Generalised } \\
\text { Linear Mixed Models }\end{array}$ \\
\hline Sex & 0.1869 & 0.7712 \\
Age (years) & $0.037^{*}$ & $0.0124^{*}$ \\
Initial cystic volume (mL) & 0.1661 & 0.1711 \\
Duration (M) & $<0.0001^{*}$ & $0.0016^{*}$ \\
Location & $0.0081^{*}$ & 0.2156 \\
Tooth extraction & 0.2628 & 0.4438 \\
Expansion of cortical layer & 0.1036 & 0.4582 \\
Pathological diagnosis & 0.2803 & 0.894 \\
\hline
\end{tabular}

* Significantly different mean percentage reduction in volume $(p<0.05)$.

There was no statistically significant difference in any other factor (sex, initial cystic volume, involved teeth, cortical bone expansion, or pathological diagnosis).

\section{Discussion}

Since cystic decompression was introduced in the conservative treatment of odontogenic cysts, several cases of treatment with decompression have been encountered, and a high success rate has been reported in various studies [9-11]. Because decompression is a long-term treatment, it is important to determine the optimal timing for cyst enucleation [3]. There are no official criteria for decompression periods or size changes [11]. After the proper removal of a lesion, a surgeon would decide on the optimal enucleation timing [5,6]. Therefore, PRV investigation of the cystic lesion is beneficial to achieve clinical success.

In previous studies, there was no disagreement about the effectiveness of volume reduction after cystic decompression. However, there are various opinions about the ideal period of cystic decompression. Marker et al. reported that it is desirable to perform cyst enucleation if the size of the cystic lesion after decompression has shrunk greater than 50\%-60\% [9]. In general, 6 to 14 months was suggested as the appropriate period of cystic decompression in most reported studies [9,10,12-18]. However, Anavi et al. reported that a long period of cystic decompression of more than 33 months in the maxilla and 22 months in the mandible before cyst enucleation was ideal [13]. Yi Zhao et al. suggested that the cystic volume would decrease significantly within 3 months after decompression 
surgery [12], and Bodner et al. reported that a minimum decompression period is required for safe cyst enucleation [19]. In summary, previous reports suggest a period of 3-14 months and a period of $50-60 \%$ reduction in size as the ideal period of decompression. The mean PRV was 54.68\%, and the mean period of decompression was 9.41 months (range, 3-27 months) in our study, which was similar to the results of the other studies.

Comparing volumetric changes in cystic lesions before and after decompression has been attempted with several analysis methods. Yi Zhao et al. obtained the cyst volume by injecting physiological saline into an empty cystic cavity and calculating the volume of the injected liquid [12]. In most studies, preoperative and postoperative panoramic radiographs or CT images were used [2,5,13,14,17,20-22]. In early days, studies utilized panoramic radiography when assessing the volumetric changes in the jaw cysts after decompression surgery [2,13]. With panoramic radiography, patients are less exposed to radiation, and it is more cost-effective than CTs. However, due to the limitation of the two-dimensional analysis of panoramic radiography, it was difficult to determine the exact volumetric changes [3]. Moreover, it is difficult to determine the exact three-dimensional association between the adjacent anatomical structures and lesions. Therefore, recently, several studies assessing the more accurate volumetric changes using 3D-CT data were been conducted [1,3,23]. Using 3D-CT, a relatively accurate shape of cysts can be determined by considering the variable shapes of the lesions, and accurate determination of volumetric changes after decompression is possible [1,3]. Furthermore, in our study, using the concept of the integral, a more precise and clearer study could be conducted using 3D-CT and a software.

The authors have previously suggested factors that affect graft failure during enucleation [24]. Graft failure with concomitant cyst enucleation could increase in cases of younger age, smoking, preoperative infection, large size, impaction of the mandibular third molar, perilesional osteosclerosis, and the use of mixed non-autogenous and autogenous bone [24]. In this study on decompression, it was confirmed that cases of long period, in younger patients, and in the posterior maxilla showed more volume reduction effect.

After the parameters had been evaluated in terms of its effect on decompression, age was considered statistically significant $(p<0.05)$. The effects of decompression based on age are controversial. Although one study reported that younger patients had a higher PRV [13], another study reported that decompression is not associated with age [25].

Longer cystic decompression periods were associated with significantly greater effectiveness of decompression $(p<0.05)$ in our study. Most previous studies were designed without considering the cystic reduction velocity during decompression, whereas some were designed considering cystic decompression velocity. Some studies showed that the volume reduction rate was higher during the initial decompression period, implying that the rate of reduction varies from with time during the period of cystic decompression $[12,20]$.

The mean PRVs were $67.3 \%, 53.9 \%$, and $52.0 \%$ in the posterior maxilla, anterior maxilla, and mandible, respectively, in our study, showing statistically significant differences $(p<0.05)$. The outcome of decompression revealed that the PRV was more favourable in the posterior maxilla, with maxillary sinus involvement, than the anterior maxilla or mandible. Jeong et al. reported that the PRV in the maxilla is significantly favourable because of the maxillary sinus, which contains several air spaces [3].

In our study, initial cystic volume was considered insignificant. Jeong et al. reported that the rate of reduction of volume was associated with the initial size of the lesion-specifically, with the group with large initial volume lesions [3]. Song et al. [21] also reported that the rate of reduction of volume was associated with the original size of the lesion [1]. However, Anavi et al. reported that the initial size did not affect the outcome of decompression [13].

The effects of the other parameters were unclear. In this study, there were no statistically significant differences in terms of tooth extraction and the rate of expansion of the cortical layer. These results were consistent to the results of the study conducted by Jeong et al. [3]. Pathological diagnosis did not influence the effect of decompression in our study, as also reported in the study by Anavi et al. [13]. However, in several studies, decompression of OKC showed more favourable results than in other types of cysts $[7,26,27]$. 
Considering that this study had a retrospective study design, the timing of CT scans and follow-up duration were inconsistent. Although we found that decompression was associated with young age, duration, and location of lesion, considering the limitation of this study, more accurate and precise clinical guidelines are required. Selective application of decompression surgery in jaw cystic lesions is a good choice to achieve an optimal therapeutic effect.

\section{Conclusions}

The conclusions of this study are as follows.

1. In the patient group to which the decompression procedure was applied, an average of $54.68 \%$ reduction in cyst size was observed during an average observation period of 9.41 months.

2. Decompression is an effective procedure for reducing the size of cysts in all patients, as determined by 3D volumetric analysis.

3. Decompression was more effective when the procedure was applied for a long period, in younger patients, and in the posterior maxilla.

Author Contributions: Conceptualisation, H.-K.L. and E.-S.L.; data curation, Y.-J.K.; formal analysis, K.-S.K. and B.-K.S.; funding acquisition, H.-K.L.; investigation, K.-S.K. and B.-K.S.; methodology, H.-K.L. and E.-S.L.; project administration, D.-H.K.; resources, H.-K.L., and Y.-J.K.; supervision, H.-K.L. and E.-S.L.; validation, H.-S.J.; visualisation, S.-H.K.; writing - original draft, Y.-J.K.; writing-review and editing, H.-K.L. and Y.-J.K. All authors have read and agreed to the published version of the manuscript.

Funding: This research was supported by the Korea University Guro Hospital “KOREA RESEARCH-DRIVEN HOSPITALS" Grant. (O1801551).

Acknowledgments: Special thanks to Soon-Young Hwang for the statistical analysis.

Conflicts of Interest: The authors declare no conflict of interest.

\section{References}

1. Asutay, F.; Atalay, Y.; Turamanlar, O.; Horata, E.; Burdurlu, M.C. Three-Dimensional Volumetric Assessment of the Effect of Decompression on Large Mandibular Odontogenic Cystic Lesions. J. Oral Maxillofac. Surg. 2016, 74, 1159-1166. [CrossRef] [PubMed]

2. Marin, S.; Kirnbauer, B.; Rugani, P.; Mellacher, A.; Payer, M.; Jakse, N. The effectiveness of decompression as initial treatment for jaw cysts: A 10-year retrospective study. Med. Oral Patol. Oral Cir. Bucal 2019, 24, e47-e52. [CrossRef] [PubMed]

3. Jeong, H.G.; Hwang, J.J.; Lee, S.H.; Nam, W. Effect of decompression for patients with various jaw cysts based on a three-dimensional computed tomography analysis. Oral Surg. Oral Med. Oral Pathol. Oral Radiol. 2017, 123, 445-452. [CrossRef] [PubMed]

4. Schlieve, T.; Miloro, M.; Kolokythas, A. Does Decompression of Odontogenic Cysts and Cystlike Lesions Change the Histologic Diagnosis? J. Oral Maxillofac. Surg. 2014, 72, 1094-1095. [CrossRef]

5. Park, H.S.; Song, I.S.; Seo, B.M.; Lee, J.H.; Kim, M.J. The Effectiveness of Decompression for Patients with Dentigerous Cysts, Keratocystic Odontogenic Tumors, and Unicystic Ameloblastoma. J. Korean Assoc. Oral Maxillofac. Surg. 2014, 40, 260-265. [CrossRef]

6. de Molon, R.S.; Verzola, M.H.; Pires, L.C.; Mascarenhas, V.I.; da Silva, R.B.; Cirelli, J.A.; Barbeiro, R.H. Five years follow-up of a keratocyst odontogenic tumor treated by marsupialization and enucleation: A case report and literature review. Contemp Clin. Dent. 2015, 6 (Suppl. 1), S106-S110. [CrossRef]

7. Nakamura, N.; Mitsuyasu, T.; Mitsuyasu, Y.; Taketomi, T.; Higuchi, Y.; Ohishi, M. Marsupialization for odontogenic keratocysts: Long-term follow-up analysis of the effects and changes in growth characteristics. Oral Surg. Oral Med. Oral Pathol. Oral Radiol. Endod. 2002, 94, 543-553. [CrossRef]

8. Schmidt, B.L.; Pogrel, M.A. The Use of Enucleation and Liquid Nitrogen Cryotherapy in the Management of Odontogenic Keratocysts. J. Oral Maxillofac. Surg. 2001, 59, 720-725. [CrossRef]

9. Marker, P.; Brondum, N.; Clausen, P.P.; Bastian, H.L. Treatment of large odontogenic keratocysts by decompression and later cystectomy: A long-term follow-up and a histologic study of 23 cases. Oral Surg. Oral Med. Oral Pathol. Oral Radiol. Endod. 1996, 82, 122-131. [CrossRef] 
10. Enislidis, G.; Fock, N.; Sulzbacher, I.; Ewers, R. Conservative Treatment of Large Cystic Lesions of the Mandible: A Prospective Study of the Effect of Decompression. Br. J. Oral Maxillofac. Surg. 2004, 42, 546-550. [CrossRef]

11. Lee, S.T.; Kim, S.G.; Moon, S.Y.; Oh, J.S.; You, J.S.; Kim, J.S. The Effect of Decompression as Treatment of the Cysts in the Jaws: Retrospective Analysis. J. Korean Assoc. Oral Maxillofac. Surg. 2017, 43, 83-87. [CrossRef] [PubMed]

12. Zhao, Y.; Liu, B.; Han, Q.B.; Wang, S.P.; Wang, Y.N. Changes in Bone Density and Cyst Volume After Marsupialization of Mandibular Odontogenic Keratocysts (Keratocystic Odontogenic Tumors). J. Oral Maxillofac. Surg. 2011, 69, 1361-1366. [CrossRef] [PubMed]

13. Anavi, Y.; Gal, G.; Miron, H.; Calderon, S.; Allon, D.M. Decompression of odontogenic cystic lesions: Clinical long-term study of 73 cases. Oral Surg. Oral Med. Oral Pathol. Oral Radiol. Endod. 2011, 112, 164-169. [CrossRef] [PubMed]

14. Zhao, Y.F.; Wei, J.X.; Wang, S.P. Treatment of odontogenic keratocysts: A follow-up of 255 Chinese patients. Oral Surg. Oral Med. Oral Pathol. Oral Radiol. Endod. 2002, 94, 151-156. [CrossRef] [PubMed]

15. Maurette, P.E.; Jorge, J.; de Moraes, M. Conservative Treatment Protocol of Odontogenic Keratocyst: A Preliminary Study. J. Oral Maxillofac. Surg. 2006, 64, 379-383. [CrossRef] [PubMed]

16. August, M.; Faquin, W.C.; Troulis, M.J.; Kaban, L.B. Dedifferentiation of Odontogenic Keratocyst Epithelium After Cyst Decompression. J. Oral Maxillofac. Surg. 2003, 61, 678-683. [CrossRef]

17. Brondum, N.; Jensen, V.J. Recurrence of keratocysts and decompression treatment. A long-term follow-up of forty-four cases. Oral Surg. Oral Med. Oral Pathol. 1991, 72, 265-269. [CrossRef]

18. Nomura, Y.; Watanabe, H.; Shirotsu, K.; Honda, E.; Sumi, Y.; Kurabayshi, T. Stability of Voxel Values from Cone-Beam Computed Tomography for Dental Use in Evaluating Bone Mineral Content. Clin. Oral Implants Res. 2013, 24, 543-548. [CrossRef]

19. Bodner, L.; Bar-Ziv, J. Characteristics of bone formation following marsupialization of jaw cysts. Dentomaxillofac. Radiol. 1998, 27, 166-171. [CrossRef]

20. Park, J.H.; Kwak, E.J.; You, K.S.; Jung, Y.S.; Jung, H.D. Volume change pattern of decompression of mandibular odontogenic keratocyst. Maxillofac. Plast Reconst. Surg. 2019, 41, 2. [CrossRef]

21. Song, I.S.; Park, H.S.; Seo, B.M.; Lee, J.H.; Kim, M.J. Effect of Decompression on Cystic Lesions of the Mandible: 3-Dimensional Volumetric Analysis. Br. J. Oral Maxillofac. Surg. 2015, 53, 841-848. [CrossRef] [PubMed]

22. Yoshikawa, Y.; Nakajima, T.; Kaneshiro, S.; Sakaguchi, M. Effective Treatment of the Postoperative Maxillary Cyst by Marsupialization. J. Oral Maxillofac. Surg. 1982, 40, 487-491. [CrossRef]

23. Rothe, J.H.; Steffen, I.G.; Lehmkuhl, L.; Grieser, C.; Mussler, A.; Schnapauff, D.; Stelter, L.; Denecke, T. Volume measurement of liver metastases using multidetector computed tomography: Comparison of lesion diameter and volume segmentation-A phantom study. Rofo 2010, 182, 1082-1090. [CrossRef] [PubMed]

24. Lim, H.K.; Kim, J.W.; Lee, U.L.; Kim, J.W.; Lee, H. Risk Factor Analysis of Graft Failure With Concomitant Cyst Enucleation of the Jaw Bone: A Retrospective Multicenter Study. J. Oral Maxillofac. Surg Off. J. Am. Assoc. Oral Maxillofac. Surg. 2017, 75, 1668-1678. [CrossRef] [PubMed]

25. Rao, S.; Rao, S. Decompression as a Treatment for Odontogenic Cystic Lesions of the Jaw. J. Oral Maxillofac. Surg. 2014, 72, 1231. [CrossRef] [PubMed]

26. Nakamura, N.; Higuchi, Y.; Tashiro, H.; Ohishi, M. Marsupialization of Cystic Ameloblastoma: A Clinical and Histopathologic Study of the Growth Characteristics Before and After Marsupialization. J. Oral Maxillofac. Surg. 1995, 53, 748-754. [CrossRef]

27. Pogrel, M.A. Treatment of Keratocysts: The Case for Decompression and Marsupialization. J. Oral Maxillofac. Surg. 2005, 63, 1667-1673. [CrossRef] [PubMed]

Publisher's Note: MDPI stays neutral with regard to jurisdictional claims in published maps and institutional affiliations. 